\title{
To Treat or not to Treat: The Case of Tuberculosis
}

\author{
Carlos Castillo-Chavez and Zhilan Feng \\ Biometrics Unit \\ Cornell University \\ Ithaca, NY 14853
}

May 15, 1995

\begin{abstract}
Incomplete treatment of patients with infectious tuberculosis (TB) may not only lead to relapse but also to the development of antibiotic resistant TB - one of the most serious health problems facing society today. In this article, we formulate one-strain and two-strain TB models to determine possible mechanisms that may allow for the survival and spread of naturally resistant strains of $\mathrm{TB}$ as well as antibiotic-generated resistant strains of TB. Analysis of our models shows that non-antibiotic co-existence is possible but rare for naturally resistant strains while co-existence is almost the rule for strains that result from the lack of compliance with antibiotic treatment by TB infected individuals.
\end{abstract}

Key words: Tuberculosis, Antibiotic Resistance, Epidemiology, Co-existence, Dynamical systems

$\mathrm{BU}-1288-\mathrm{M}$

May 1995 


\section{Introduction}

Infectious diseases like measles, influenza, chicken pox, and rubeola have several features in common; for example, they cause recurrent epidemic outbreaks and transmission rates depend strongly on age-dependent contact rates. The etiological agents of these communicable diseases are viruses from different families but all capable of generating similar epidemiological responses at the level of the individual (symptoms). Common responses include relatively short latent periods, followed by also relatively short infectious periods and permanent immunity after recovery. It is not completely clear when individuals become infectious (that is, capable of transmitting the disease) as some may become infectious while symptomless. Effective vaccines have been developed for these communicable diseases (to some degree only influenza remains the major challenge, as the family of viruses responsible for the "flu" experiences continuous minor and major genetic changes). A dense and mature literature associated with the use of mathematical models to study communicable diseases such as measles, influenza, rubeola, and chicken pox is already in place (see Hethcote 1976, Dietz 1979, Hethcote, Stech, and van den Driescsche 1981, Anderson 1982, Anderson and May 1982, 1991, Dietz and Schenzle 1985, Dietz 1985, Anderson and May 1983, Schenzle 1984, Hethcote and Van Ark 1987, Castillo-Chavez et al. 1988, 1989, Feng 1994, Feng and Thieme 1995). The situation of tuberculosis, despite its fundamental role in the development of bacteriology and modern epidemiology, is paradoxically different.

Tuberculosis (TB) is a bacterial disease with about one third of the world human population as its reservoir (Bloom 1994, Miller 1993). It is one of the oldest recorded human diseases (it seems clear that TB has afflicted animal populations before the origin of the human species). Evidence that supports human cases of TB as well as its role in human mortality goes back for centuries (petrified bones 8,000 B.C., Hindu texts from 2,000 B.C. and mummified reliquiae from Egypt and pre-Columbian America including an Incan child 700 A.D.). TB was so devastating that it became the motivating force in the development of the fields of bacteriology, modern epidemiology, and public health. TB or TB associated symptoms appear to have been the source of inspiration for Frascatorius' theory of contagion (18th century). However, a search for a cause without a clear understanding of the sources and nature of disease, naturally led to what Ayvazain (Ayvazain 1993) calls "centuries of nonscientific chaos."

The situation changed when Villeman (19th century) used animal models to establish

TB as a specific infection due to an innoculable agent (Reichman and Hershfield 1993). On March 29, 1882, Robert Koch presented to the Berlin Physiologic Society the results of his research on the causes of disease. Koch's fundamental research identified the mechanisms for disease transmission and the agents responsible for some diseases, including the etiological agent of TB (Reichman and Hershfield 1993). Koch's research opened new doors and eventually led to the discovery by various investigators of other bacteriological disease 
agents including the bacilli for typhoid, glanders, and diphteria.

Despite its sociological and historical importance, the study of the spread of TB using statistical and mathematical models has not received enough attention. In fact we have observed only an extremely limited use of mathematical models in the study of the transmission dynamics of TB in human populations (see Blower 1995 and references therein). Tuberculosis is caused by Mycobacterium tuberculosis. The disease is most commonly transmitted from a person suffering from infectious (active) tuberculosis to other persons by infected droplets created when the person with active TB coughs or sneezes. Among generally healthy persons, infection with TB is highly likely to be asymptomatic. Data from a variety of sources suggest that the life time risk of developing clinically evident TB after being infected is approximately $10 \%$, with $90 \%$ likelihood of the infection remaining latent (Hopewell 1994). Individuals who have a latent TB infection are not clinically ill nor capable of transmitting TB (Miller 1993). At greater ages, the immunity of persons who have been previously infected may wane, and they may be then at risk of developing active TB as a consequence of either exogenous reinfection (i.e., acquiring a new infection from another infectious individual) or endogenous reactivation of latent bacilli (i.e., re-activation of a pre-existing dormant infection) (Styblo 1991, Smith 1994).

The epidemiology of TB disease is not simple. For the purpose of this article we only provide a superficial view which we believe is sufficient for a rough understanding of the dynamics of TB transmission at the population level. General sources of information on TB dynamics suggest that TB is hard to transmit. Transmission (it is said) occurs only when there is prolonged close contact between a susceptible person and a person who has an active case of TB. Nonetheless, under the right conditions a single person with active TB can infect many other people (Salyers 1994). For example, it seems that about 13 persons were infected with TB per year by one source of infection in a Netherlands community in the period 1921-1938 (Styblo 1991). However, it is not clear that TB is in fact hard to transmit. Recent documented cases of TB transmission during lengthy plane trips (Kolata 1995, MMWR 1995) seem to indicate that transmission may be highly facilitated in a modern society. It is not at all unlikely that the risk of infection may be quite high in public places where there are actively infected TB individuals present. Recently mathematical models have been developed to estimate the probability of transmission of $\mathrm{TB}$ in close public environments. These models support the view that the acquisition of TB infection may not be as difficult as previously thought (Edward Nardell 1995). A naive look at the fact that one third of the world population is actually infected suggests that either the tubercle bacillus is easy to acquire, or that in many parts of the world exposure and re-exposure to TB is extremely persistent, or both. Current epidemiological studies strongly support the claim that exposed individuals are unable to transmit the tubercle bacillus and that only individuals with "active" TB are capable of spreading this 
bacteria. Therefore, exposed individuals provide a tremendously large reservoir for the tubercle bacillus but as latent carriers of this bacillus they are uncapable of transmission. What are the epidemiological consequences of this situation in a world where populations become closer and closer? Here lies one of the central issues associated with the study of TB dynamics.

Exposed TB individuals may remain in this latent stage for variable periods of time (in fact, many die without ever developing active TB). Apparently, the longer that we carry this bacteria the less likely we are to develop active TB unless our immune system becomes seriously compromised by other diseases. Consequently, age of infection as well as chronological age are important factors in disease progression. How important are these factors as predictors or measures of spread at the population level? Because it has been estimated that $10 \%$ of those infected with TB actually develop active TB during their life time then the $10 \%$ rule has become a useful measure for rough and immediate public health measures. This rule is useful but at the same time it is also superficial. It is well known that TB progression is not uniform but in fact is closely linked to various other factors such as nutritional status and/or access to decent medical care and living conditions (Bloom 1994). The good news is that latent and active TB can be treated with antibiotics. The bad news is that its treatment has side effects (sometimes quite serious) and takes a long time. Carriers of the tubercle bacillus who have not developed TB disease can be treated with a single drug $I N H$; unfortunately, it must be taken religiously for 6-9 months. Treatment for those with active TB requires the simultaneous use of three drugs for a period of at least 12 months. Lack of compliance with these drug treatments (a very serious problem) not only may lead to a relapse but to the development of antibiotic resitant TB - one of the most serious public health problems facing society today.

TB remains the leading cause of death by an infectious disease in the world. TB is also the most prevalent infection in the world (Bloom 1994, Miller 1993). As stated before, a third of the world's population is a carrier of tuberculosis and is at risk for developing active TB. It is estimated that there are between 8 and 10 million new cases per year, of which about 3 million people die (Kochi 1991). In the United States, the estimated total number of TB infections lies between 10-15 million persons (Miller 1993). However, dramatic increases in the incidence of TB (new cases per year) have occurred within the United States over the past few years. From 1985 to 1991, the number of reported cases of TB has increased $18 \%$ with 26,283 cases reported in 1991 (Kent 1993). In 1991, a large California prison with 5,421 inmates and 1,500 staff members had 18 cases of active TB (Salyers 1994). Against the backdrop of an increasing incidence of TB in the United States there is a second problem, namely that of multi-drug resistant TB (MDR-TB). ResistantTB develops when the treatment of a TB patient is inadequate or incomplete, thereby allowing some of the stronger/resistant bacilli to survive and prosper. Outbreaks of MDR- 
TB in the United States have begun to alarm doctors and public health officials. Over $80 \%$ of the patients in these outbreaks have died, often within weeks of being diagnosed as having tuberculosis. These problems are compounded by economics, as the cost of treating a patient with MDR-TB can exceed $\$ 250,000$ : nearly 100 times the cost of treating most other TB cases (Press release WHO /89 Nov. 1994). The emergence of the HIV epidemic has dramatically increased the risk of developing clinical TB in infected persons, substantially increasing TB rates globally (Miller 1993).

A TB vaccine called BCG (Bacillus of Calmette and Guérin) has been available for many decades. The BCG vaccine is cheap, costing about 10 cents per dose but its effectiveness in preventing TB is controversial (Salyers 1994). Results of field trials of the vaccine have differed widely, some indicating protection rates as high as $70 \%$ to $80 \%$, others indicating the vaccine was completely ineffective in preventing TB (Salyers 1994). Potential problems associated with the generalized use of the BCG vaccine in some populations are closely associated to the fact that vaccinated individuals will test positive for TB. It becomes therefore nearly impossible to be able to detect the prevalence of a disease in a population (like the Argentinian population) where most individuals are vaccinated.

The purposes of this paper are quite specific. We formulate a basic transmission model to study the dynamics of TB in as simple a setting as possible. The advantage of this approach is that using this simple setting, we are able to fully analyze the effects of basic epidemiological factors such as the latent and infectious periods on the dynamics of TB on a homogeneosuly mixing population. This model then becomes our basic structure to begin the study of the effects of resistant-TB on the same population. The difficulties in treating these infectious patients and their role in spreading drug-resistant bacilli to others is incorporated in our model.

This paper is organized as follows: Section 1 introduces a "simple" TB model. We compute its basic reproductive number and study its role on the dynamics and stability properties of this model. In Section 2 we introduce a two-strain TB model and study its dynamics under two distinct assumptions. First, we assume that we are only dealing with two competing strains and we find that co-existence is possible but "rare". Secondly we assume that the second strain is the result of antibiotic resitance and find that co-existence is common. Our mathematical results are based on quasi-steady state approximations and hence our analysis is not complete. Section 3 is devoted to the conduction of numerical simulations that support or complete our analytical results. Our simulations involve the construction of bifurcation diagrams that support the results of Section 2. Section 4 det ails some of our current efforts and extensions including the incorporation of distributed delays, re-infection, and the effects of age-dependent contact rates. An appendix collects some of the mathematical details. 


\section{A simple TB model}

In this section we introduce a simple model for the transmission of TB. The host population is divided into the following epidemiological class or subgroups: Susceptibles $(S)$, Latent ( $L$, infected but not infectious), Infectious $(I)$, and (effectively) Treated $(T)$ individuals. $N$ denotes the total population. Using Fig. 1.a we formulate the following model for TB:

$$
\begin{aligned}
\frac{d}{d t} S & =\Lambda-\beta c S \frac{I}{N}-\mu S \\
\frac{d}{d t} L & =\beta c S \frac{I}{N}-\left(\mu+k+r_{1}\right) L+\beta^{\prime} c T \frac{I}{N} \\
\frac{d}{d t} I & =k L-(\mu+d) I-r_{2} I \\
\frac{d}{d t} T & =r_{1} L+r_{2} I-\beta^{\prime} c T \frac{I}{N}-\mu T \\
N & =S+L+I+T .
\end{aligned}
$$

$\Lambda$ is the recruitment rate, $\beta$ and $\beta^{\prime}$ are the average proportions of susceptible and treated individuals infected by one infectious individual per contact per unit of time, respectively, $c$ is the per-capita contact rate, $\mu$ is the per-capita natural death rate, $k$ is the rate at which an individual leaves the latent class by becoming infectious, $d$ is the per-capita disease induced death rate, and $r_{1}$ and $r_{2}$ are per-capita treatment rates. We assumed that an individual may be infected only through contacts with infectious individuals.

It can be shown that for System (1.1) the first octant in the state space is positively invariant. Adding equations in (1.1) gives

$$
\frac{d}{d t} N=\Lambda-\mu N-d I
$$

Note that $\frac{d}{d t} N(t)<0$ for $N>\Lambda / \mu$. Hence, without loss of generality, we can consider only solutions of (1.1) on the following positively invariant subset of $\mathbf{R}^{4}$ :

$$
G=\left\{(S, L, I, T) \mid S, L, I, T \geq 0, S+L+I+T \leq \frac{\Lambda}{\mu}\right\} .
$$

The basic reproductive number for (1.1) is

$$
\mathcal{R}_{0}=\left(\frac{\beta c}{\mu+d+r_{2}}\right)\left(\frac{k}{\mu+k+r_{1}}\right)
$$

that is, the basic reproductive number is given by the product $\beta c /\left(\mu+r_{2}+d\right)$, the average number of susceptibles infected by one infectious individual during his or her effective 
infectious period and $k /\left(\mu+r_{1}+k\right)$, the fraction of the population which survives the latent period. Therefore $\mathcal{R}_{0}$ gives the number of secondary infectious cases produced by an infectious individual during his or her effective infectious period when introduced in a population of susceptibles.

If we assume that the infection probabilities per contact for the treated class is the same as that of the susceptible class, i.e., $\beta^{\prime}=\beta$, then System (1.1) can be reduced to the following 3-D system:

$$
\begin{aligned}
\frac{d}{d t} W & =\Lambda-\beta c W \frac{I}{N}-\mu W+r_{1} L+r_{2} I \\
\frac{d}{d t} L & =\beta c W \frac{I}{N}-\left(\mu+k+r_{1}\right) L \\
\frac{d}{d t} I & =k L-\left(\mu+d+r_{2}\right) I \\
N & =W+L+I,
\end{aligned}
$$

where $W=S+T$. Replacing the $\frac{d}{d t} W$ equation in System (1.3) by the $\frac{d}{d t} N$ equation given by (1.2) leads to the the following equivalent system:

$$
\begin{aligned}
\frac{d}{d t} N & =\Lambda-\mu N-d I \\
\frac{d}{d t} L & =\beta c(N-L-I) \frac{I}{N}-\left(\mu+k+r_{1}\right) L \\
\frac{d}{d t} I & =k L-\left(\mu+d+r_{2}\right) I .
\end{aligned}
$$

System (1.4) may have two equilibria given by the disease-free equilibrium

$$
E^{0}=\left(N^{0}, L^{0}, I^{0}\right)=\left(\frac{\Lambda}{\mu}, 0,0\right),
$$

and if $\mathcal{R}_{0}>1$, the unique endemic equilibrium is

$$
E^{*}=\left(N^{*}, L^{*}, I^{*}\right)
$$

where

$$
\begin{aligned}
N^{*} & =\frac{\alpha \mathcal{R}_{0} \Lambda}{d k\left(\mathcal{R}_{0}-1\right)+\mu \alpha \mathcal{R}_{0}} \\
L^{*} & =\frac{\mu+d+r_{2}}{k} I^{*} \\
I^{*} & =\frac{k\left(\mathcal{R}_{0}-1\right)}{\alpha \mathcal{R}_{0}} N^{*},
\end{aligned}
$$

and

$$
\alpha=\mu+d+r_{2}+k
$$


We have established the following result on the dynamics of System (1.4).

Theorem 1. If $\mathcal{R}_{0}<1$ then the disease-free equilibrium $E^{0}$ is globally asymptotically stable (g.a.s.). While if $\mathcal{R}_{0}>1$ then the unique endemic equilibrium $E^{*}$ is locally asymptotically stable (1.a.s.).

The proof is given in the Appendix.

If there is no treatment, that is, if $r_{1}=r_{2}=0$ in System (1.1), then the qualitative dynamics are identical to those of the model with positive treatment rates. However, because $\mathcal{R}_{0}$ is a decreasing function of $r_{1}$ and $r_{2}$, and because the value of $\mathcal{R}_{0}$ decreases when we increase the treatment rates, then the disease levels (quantitative dynamics) are different, that is, treatment -as expected - reduces prevalence while increasing the fraction of non-infected population $\left(S^{*}+T^{*}\right) / N^{*}$. 


\section{A two strain TB model}

The increasing recent number of outbreaks of active TB signal the creation of new opportunities for the development of resistant strains. In this section we modify our earlier model (System (1.1)) to take into account the possible appearance of resistant strains due to the deficient compliance with treatment schedules. We add two additional classes $L_{2}$ (latent) and $J$ (infectious) representing the developmental stages of resistant strains. Furthermore, we assume that $J$ individuals can infect $S, L_{1}$, and $T$ individuals. From the disease transmission diagram (see Fig. 2.b) we can write the following system of ordinary differential equations:

$$
\begin{aligned}
\frac{d}{d t} S & =\Lambda-\beta c S \frac{I}{N}-\beta^{*} c S \frac{J}{N}-\mu S \\
\frac{d}{d t} L_{1} & =\beta c S \frac{I}{N}-(\mu+k) L_{1}-r_{1} L_{1}+p r_{2} I+\beta^{\prime} c T \frac{I}{N}-\beta^{*} c L_{1} \frac{J}{N} \\
\frac{d}{d t} I & =k L_{1}-(\mu+d) I-r_{2} I \\
\frac{d}{d t} T & =r_{1} L_{1}+(1-p-q) r_{2} I-\beta^{\prime} c T \frac{I}{N}-\beta^{*} c T \frac{J}{N}-\mu T \\
\frac{d}{d t} L_{2} & =q r_{2} I-\left(\mu+k^{\prime}\right) L_{2}+\beta^{*} c\left(S+L_{1}+T\right) \frac{J}{N} \\
\frac{d}{d t} J & =k^{\prime} L_{2}-\left(\mu+d^{\prime}\right) J \\
N & =S+L_{1}+I+T+L_{2}+J
\end{aligned}
$$

where $\beta^{*}$ is the average proportion of individuals infected by one resistant-TB infectious individual per contact per unit of time; $d^{\prime}$ and $k^{\prime}$ have similar meanings as $d$ and $k ; p+q$ is the proportion of those treated infectious individuals who did not complete their treatment. The proportion $p$ modifies the rate that departs from the latent class; $q r_{2} I$ gives the rate at which individuals develop resistant-TB because they did not complete the treatment of active TB. Therefore $p \geq 0, q \geq 0$ and $p+q \leq 1$.

For System (2.1) the first octant in the state space is positively invariant. By adding the equations in (2.1) we get the equation for $\frac{d}{d t} N$ :

$$
\frac{d}{d t} N=\Lambda-\mu N-d I-d^{\prime} J .
$$

Since $\frac{d}{d t} N(t)<0$ for $N>\Lambda / \mu$, all solutions of (2.1) with nonnegative initial data approach, enter, or stay inside the subset $\Omega$ defined by $0 \leq S+L_{1}+I+T+L_{2}+J \leq \Lambda / \mu$. Hence, without loss of generality, we can only consider solutions of (2.1) on $\Omega$. Using Lemma 1 found in the Appendix we get the inequality

$$
N_{\infty} \geq \frac{\Lambda}{\mu+d+d^{\prime}} .
$$


Since the right hand side of (2.1) is continuously differentiable there exists a unique solution on a maximal forward time interval for any nonnegative initial data, and hence the initial value problem (2.1) with initial data in $\Omega$ is well posed.

The basic reproductive numbers for the two-strain model are given by

$$
\mathcal{R}_{1}=\left(\frac{\beta c+p r_{2}}{\mu+d+r_{2}}\right)\left(\frac{k}{\mu+k+r_{1}}\right)
$$

and

$$
\mathcal{R}_{2}=\left(\frac{\beta^{*} c}{\mu+d^{\prime}}\right)\left(\frac{k^{\prime}}{\mu+k^{\prime}}\right),
$$

respectively. We can interpret $\mathcal{R}_{1}$ and $\mathcal{R}_{2}$ as the average numbers of secondary infectious cases produced by an ordinary TB strain and one resistant-TB infectious individual during his or her effective infectious period, respectively.

If we let

$$
\mathcal{R}_{0}=\max \left\{\mathcal{R}_{1}, \mathcal{R}_{2}\right\}
$$

then $\mathcal{R}_{0}=1$ gives a threshold condition in the sense that the disease will die out if $\mathcal{R}_{0}<1$ while the disease may become endemic if $\mathcal{R}_{0}>1$.

Next we consider the case $\beta^{\prime}=\beta$. We again let $W=S+T$, hence

$$
\frac{d}{d t} W=\Lambda-\beta c W \frac{I}{N}-\mu W+r_{1} L_{1}+(1-p-q) r_{2} I-\beta^{*} c W \frac{J}{N} .
$$

An equivalent system to (2.1) when $\beta^{\prime}=\beta$ is given by (2.3) and

$$
\begin{aligned}
\frac{d}{d t} L_{1} & =\beta c W \frac{I}{N}-(\mu+k) L_{1}-r_{1} L_{1}+p r_{2} I-\beta^{*} c L_{1} \frac{J}{N}, \\
\frac{d}{d t} I & =k L_{1}-(\mu+d) I-r_{2} I \\
\frac{d}{d t} L_{2} & =q r_{2} I-\left(\mu+k^{\prime}\right) L_{2}+\beta^{*} c\left(W+L_{1}\right) \frac{J}{N} \\
\frac{d}{d t} J & =k^{\prime} L_{2}-\left(\mu+d^{\prime}\right) J
\end{aligned}
$$

where

$$
N=W+L_{1}+I+L_{2}+J .
$$

If we replace the equation for $\frac{d}{d t} W$ in (2.3) by (2.2) we get the following equivalent system:

$$
\begin{aligned}
\frac{d}{d t} N & =\Lambda-\mu N-d I-d^{\prime} J, \\
\frac{d}{d t} L_{1} & =\beta c\left(N-L_{1}-I-L_{2}-J\right) \frac{I}{N}-(\mu+k) L_{1}-r_{1} L_{1}+p r_{2} I-\beta^{*} c L_{1} \frac{J}{N}, \\
\frac{d}{d t} I & =k L_{1}-(\mu+d) I-r_{2} I, \\
\frac{d}{d t} L_{2} & =q r_{2} I-\left(\mu+k^{\prime}\right) L_{2}+\beta^{*} c\left(N-I-L_{2}-J\right) \frac{J}{N}, \\
\frac{d}{d t} J & =k^{\prime} L_{2}-\left(\mu+d^{\prime}\right) J .
\end{aligned}
$$


To simplify future expressions we introduce the following notations:

$$
\begin{aligned}
R_{1 a} & =\frac{\beta c}{\mu+k+r_{1}}, \\
R_{1 b} & =\frac{k}{\mu+d+r_{2}}, \\
R_{2 a} & =\frac{k^{\prime}}{\mu+d^{\prime}} \\
q_{1 a} & =\frac{\mu}{\mu+k+r_{1}} .
\end{aligned}
$$

When $q \neq 0$, the system (2.5) has three equilibria $E_{i}, i=1,2,3$. (In this case there is no boundary equilibrium where only the first strain is present.) $E_{1}$ is the disease-free equilibrium

$$
E_{1}=\left(\frac{\Lambda}{\mu}, 0,0,0,0\right)
$$

$E_{2}$ describes the case where only the second strain is present, that is,

$$
E_{2}=\left(N_{2}, 0,0,\left(\mu+d^{\prime}\right) m\left(1-\frac{1}{\mathcal{R}_{2}}\right) N_{2}, k^{\prime} m\left(1-\frac{1}{\mathcal{R}_{2}}\right) N_{2}\right),
$$

where

$$
\begin{aligned}
N_{2} & =\frac{\Lambda}{\mu+d^{\prime} k^{\prime} m\left(1-\frac{1}{\mathcal{R}_{2}}\right)}, \\
m & =\frac{1}{\mu+d^{\prime}+k^{\prime}} .
\end{aligned}
$$

$E_{2}$ exists only when $\mathcal{R}_{2}>1$ (for any $\mathcal{R}_{1}>0$ ). $E_{3}$ describes the case where both strains are present:

$$
E_{3}=\left(N^{*}, L_{1}^{*}, I^{*}, L_{2}^{*}, J^{*}\right) .
$$

The region where $E_{3}$ exists is described explicitly in the Appendix.

To solve for $E_{3}$, let

$$
u=\frac{I}{N}, \quad v=\frac{J}{N}
$$

From the third and the fifth equations in (2.5) we have

$$
\begin{aligned}
& L_{1}=\frac{\mu+d+r_{2}}{k} I, \\
& L_{2}=\frac{\mu+d^{\prime}}{k^{\prime}} J .
\end{aligned}
$$

Substituting into the second equation in (2.5) and using (2.6) we get

$$
R_{1 a}\left(1+R_{1 b}\right) u+\left(R_{1 a} R_{1 b}\left(1+\frac{1}{R_{2 a}}\right)+\frac{\beta^{*}}{\beta} R_{1 a}\right) v+1-\mathcal{R}_{1}=0
$$


This gives

$$
u=a-b v
$$

where

$$
\begin{aligned}
& a=\frac{\mathcal{R}_{1}-1}{R_{1 a}\left(1+R_{1 b}\right)}, \\
& b=\frac{R_{1 b}\left(1+\frac{1}{R_{2 a}}\right)+\frac{\beta^{*}}{\beta}}{\left(1+R_{1 b}\right)} .
\end{aligned}
$$

Substituting into the fourth equation in (2.5) we get

$$
A v^{2}+B v+C=0
$$

where

$$
\begin{aligned}
& A=1+\frac{1}{R_{2 a}}-b, \\
& B=\frac{1}{\mathcal{R}_{2}}+\frac{b q r_{2}}{\beta^{*} c}+a-1, \\
& C=-\frac{a q r_{2}}{\beta^{*} c} .
\end{aligned}
$$

The positive real roots $v^{*}$ of $(2.9)$, if there are any, are given by

$$
v^{*}=\frac{1}{2 A}\left(-B \pm \sqrt{B^{2}-4 A C}\right) .
$$

$E_{3}$ can be expressed as

$$
\begin{aligned}
N^{*} & =\frac{\Lambda}{\mu+d u^{*}+d^{\prime} v^{*}}, \\
L_{1}^{*} & =\frac{\mu+d+r_{2}}{k} u^{*} N^{*}, \\
I^{*} & =u^{*} N^{*} \\
L_{2}^{*} & =\frac{\mu+d^{\prime}}{k^{\prime}} v^{*} N^{*}, \\
J^{*} & =v^{*} N^{*},
\end{aligned}
$$

where $v^{*}, u^{*}$ are given by (2.10) and (2.7). The feasibility of $E_{3}$ will be discussed in Theorem 2.

The case $q=0$ gives the boundary equilibrium $E_{4}\left(\right.$ if $\left.\mathcal{R}_{1}>1\right)$ :

$$
E_{4}=\left(N_{4}, \frac{e\left(\mathcal{R}_{1}-1\right)}{R_{1 b}} N_{4}, e\left(\mathcal{R}_{1}-1\right) N_{4}, 0,0\right)
$$


where

$$
N_{4}=\frac{\Lambda}{\mu+d e\left(\mathcal{R}_{1}-1\right)}, \quad e=\frac{1}{R_{1 a}\left(1+R_{1 b}\right)}
$$

To conduct an analytical analysis of asymptotical behaviors of the equilibrium points we assume that there is no disease-induced death rate, i.e., $d=d^{\prime}=0$. For $d>0$ and $d^{\prime}>0$ our numerical simulations support similar results (see Section 3 ). Hence we have that

$$
\frac{d}{d t} N=\Lambda-\mu N
$$

and, consequently, $N(t) \rightarrow \Lambda / \mu$ as $t \rightarrow \infty$. Without loss of generality (see Thieme 1992, 1994) we assume that our population has reached its limiting value, i.e.,

$$
N \equiv \Lambda / \mu \equiv W+L_{1}+I+L_{2}+J
$$

By introducing the fractions

$$
x_{1}=\frac{L_{1}}{N}, \quad x_{2}=\frac{I}{N}, \quad y_{1}=\frac{L_{2}}{N}, \quad y_{2}=\frac{J}{N},
$$

and eliminating the equation for $\frac{d}{d t} N$ we obtain from (2.5) the equivalent limiting system

$$
\begin{aligned}
& \frac{d}{d t} x_{1}=\beta c\left(1-x_{1}-x_{2}-y_{1}-y_{2}\right) x_{2}-\left(\mu+k+r_{1}\right) x_{1}+p r_{2} x_{2}-\beta^{*} c x_{1} y_{2} \\
& \frac{d}{d t} x_{2}=k x_{1}-\left(\mu+r_{2}\right) x_{2} \\
& \frac{d}{d t} y_{1}=q r_{2} x_{2}-\left(\mu+k^{\prime}\right) y_{1}+\beta^{*} c\left(1-x_{2}-y_{1}-y_{2}\right) y_{2} \\
& \frac{d}{d t} y_{2}=k^{\prime} y_{1}-\mu y_{2} .
\end{aligned}
$$

Obviously

$$
0 \leq x_{1}+x_{2}+y_{1}+y_{2} \leq 1
$$

for all time $t \geq 0$. With this notation, we are able to setablish the following result:

Theorem 2 Assume that $q=0, d=d^{\prime}=0$. Then

(a). The disease-free equilibrium $E_{1}$ of System (2.5) is g.a.s. if $\mathcal{R}_{0}<1$, i.e., if $\mathcal{R}_{1}<1$ and $\mathcal{R}_{2}<1$.

(b). If $\mathcal{R}_{1}>1$, then there exists a threshold curve given by the function $f\left(\mathcal{R}_{1}\right)$ such that the boundary equilibrium $E_{4}$ of (2.5) is l.a.s. if $\mathcal{R}_{2}<f\left(\mathcal{R}_{1}\right)$ and unstable if $\mathcal{R}_{2}>f\left(\mathcal{R}_{1}\right)$. Moreover, $f\left(\mathcal{R}_{1}\right)>1$ for all $\mathcal{R}_{1}>1$ and $f(1)=1$. 
(c). If $\mathcal{R}_{2}>1$, then there exists a second threshold curve given by the function $g\left(\mathcal{R}_{1}\right)$ such that the boundary equilibrium $E_{2}$ of (2.5) is 1.a.s. if $\mathcal{R}_{1}<1$ or if $\mathcal{R}_{1}>1$ and $\mathcal{R}_{2}>g\left(\mathcal{R}_{1}\right) . E_{2}$ is unstable if $\mathcal{R}_{1}>1$ and $\mathcal{R}_{2}<g\left(\mathcal{R}_{1}\right)$. Moreover $g\left(\mathcal{R}_{1}\right)>f\left(\mathcal{R}_{1}\right)>1$ for all $\mathcal{R}_{1}>1$ and $g(1)=1$.

(d). The equilibrium $E_{3}$ of (2.5) exists if $\mathcal{R}_{1}>1$ and $f\left(\mathcal{R}_{1}\right)<\mathcal{R}_{2}<g\left(\mathcal{R}_{1}\right)$. At the quasi-steady state $\frac{d}{d t} x_{2}=\frac{d}{d t} y_{2}=0$, i.e., when $x_{2}=R_{1 b} x_{1}$ and $y_{2}=R_{2 a} y_{1}$, the corresponding positive equilibrium is l.a.s. when it exists.

The proof of Theorem 2 is given in the Appendix. Our numerical simulations suggest that $E_{3}$ is l.a.s. not only at the quasi-steady state (when the conditions in (d) are satisfied) but in general. Theorem 2 states that using $\mathcal{R}_{1}$ and $\mathcal{R}_{2}$ as parameters the existences as well as the stabilities of all possible equilibria of (2.5) can be completely determined by threshold conditions $\mathcal{R}_{0}=1, \mathcal{R}_{2}=f\left(\mathcal{R}_{1}\right)$, and $\mathcal{R}_{2}=g\left(\mathcal{R}_{1}\right)$. We also notice that for realistic parameter values the region for co-existence is very small under the assumption of Theorem 2. This means that if we assume that we are only dealing with two competing strains $(q=0)$ then co-existence is possible but "rare". Fig. 2.a gives a bifurcation diagram for the case $q=0$.

\section{Remarks:}

1. We see, from the proof of Theorem 2, that the functions $f\left(\mathcal{R}_{1}\right)$ and $g\left(\mathcal{R}_{1}\right)$ can be determined by fixing all parameter values related to the first strain except $\beta$. Hence $\mathcal{R}_{1}$ can be varied by varying $\beta$. Furthermore, since $\mathcal{R}_{2}$ is a monotone increasing function of $\beta^{*}$, the bifurcation diagram can be drawn using $\beta$ and $\beta^{*}$ instead of $\mathcal{R}_{1}$ and $\mathcal{R}_{2}$.

2. Our numerical simulations suggest that non-trivial equilibria $E_{i}(i=2,3,4)$ are g.a.s. whenever they are l.a.s..

We now consider the case $q>0$. In this case System (2.5) has only three equilibrium $E_{1}, E_{2}$ and $E_{3}$. We have established the following result:

Theorem 3 Assume that $q>0, d=d^{\prime}=0$. Then

(i). The disease-free equilibrium $E_{1}$ of (2.5) is g.a.s. if $\mathcal{R}_{0}<1$, i.e., if $\mathcal{R}_{1}<1$ and $\mathcal{R}_{2}<1$.

(ii). If $\mathcal{R}_{2}>1$, then the boundary equilibrium $E_{2}$ of (2.5) is 1.a.s. if $\mathcal{R}_{1}<1$ or if $\mathcal{R}_{1}>1$ and $\mathcal{R}_{2}>g\left(\mathcal{R}_{1}\right)$. ( $g$ is the function given in Theorem $2(c)$ ). $E_{2}$ is unstable if $\mathcal{R}_{1}>1$ and $\mathcal{R}_{2}<g\left(\mathcal{R}_{1}\right)$.

(iii). The equilibrium $E_{3}$ of (2.5) exists iff $\mathcal{R}_{1}>1$ and $\mathcal{R}_{2}<g\left(\mathcal{R}_{1}\right)$. (In this case $E_{2}$ is unstable). When it exists, $E_{3}$ is l.a.s. at the quasi-steady state $\frac{d}{d t} x_{2}=\frac{d}{d t} y_{2}=0$, i.e., when $x_{2}=R_{1 b} x_{1}$ and $y_{2}=R_{2 a} y_{1}$.

The proof of Theorem 3 can be found in the Appendix. Fig. 2.b gives a bifurcation diagram for the case $q>0$. By analyzing the parameters (also from Fig. 2) we see that 
co-existence is almost the rule under the assumption that the second strain is the result of antibiotic resistance $(q>0)$. For the case when $d>0, d^{\prime}>0$ our numerical simulations and bifurcation diagram also support similar results (see Fig. 5). 


\section{Numerical simulations}

In this section we study the system (2.5) (or (2.13) in the case $d=d^{\prime}=0$ ) numerically to confirm our analytical results as well as to provide evidences that our results are likely to hold in more general situations. First we "extend" the result of Theorem 2 (d) numerically. Our simulations support the stability of the interior equilibrium $E_{3}$ for the system (2.13) not only at the quasi-steady state but in general (see Fig. 3). Fig. 3 presents some phase portraits for the system (2.13) which show that (for parameters in certain region (see Fig. 2)) the corresponding l.a.s. equilibrium $E_{i}(i=2,3,4)$ attracts all solutions with positive initial data (see Fig. 3). Similar simulations have been carried out when $q>0$ to support the same conclusion (see Theorem 3) that the interior equilibrium $E_{3}$ is asymptotically stable whenever it exists not only at the quasi-steady state but in general, and that the non-trivial equilibrium switches stability as the parameters change as specified in the bifurcation diagram (see Fig. 2.b and Fig. 4). We also "extend" the results of Theorem 3 numerically to the case when $d>0$ and $d^{\prime}>0$. This is done by establishing a functional relationship between $\beta$ and $\beta^{\prime}$ and by showing numerically that this function plays a role similar to that of the function $g$ in Theorem 3 (see Fig. 5).

For the construction of Fig. 3 we have selected for illustration purposes the following parameter values: $\mu=0.143$ ( $1 / \mu=70$ years), $\beta=13, c=1, k=1, q=0, p=0.5, r_{1}=$ $1, r_{2}=2, k^{\prime}=1, \Lambda=35, d=d^{\prime}=0$. This choice of parameter values gives $\mathcal{R}_{1}=3.45$. Using the formula for $f\left(\mathcal{R}_{1}\right)$ and $g\left(\mathcal{R}_{1}\right)$ (see the Appendix) we get

$$
\begin{aligned}
& \left(\mathcal{R}_{1}, \mathcal{R}_{2}\right) \in I I I \quad \text { iff } 1.34<\mathcal{R}_{2}<4.13 \\
& \left(\mathcal{R}_{1}, \mathcal{R}_{2}\right) \in I I \text { iff } \mathcal{R}_{2}>4.13 \\
& \left(\mathcal{R}_{1}, \mathcal{R}_{2}\right) \in I V \text { iff } \mathcal{R}_{2}<1.34
\end{aligned}
$$

The value of $\mathcal{R}_{2}$ for Fig. 3 .a is chosen to be 2 . Our simulations show that $E_{3}$ attracts all solutions with positive initial data. Values of $\mathcal{R}_{2}$ for Fig. 3.b and Fig. 3.c: are 4.5 and 1.2, and our simulations support the global stabilities of $E_{2}$ and $E_{4}$, respectively.

Fig. 4 is for the case when $q>0$, and $d=d^{\prime}=0$. The parameter values used in Fig. 4 are $k=0.5, k^{\prime}=1, \mu=0.0143, q=0.01, p=0.4, r_{1}=2, r_{2}=1, d=0, d^{\prime}=0, \Lambda=500$. $\bar{\beta}$ is chosen to be 13 as it corresponds to $\overline{\mathcal{R}}_{1}=2.627$ and $\overline{\mathcal{R}}_{2}=2.7364$. This last selection implies that $E_{2}$ is l.a.s. if $\mathcal{R}_{2}<2.7364$ and it also implies that $E_{3}$ exists and is 1.a.s. if $\mathcal{R}_{2}>2.7364$. In Fig. 4 , the values for $\mathcal{R}_{2}$ are chosen to be (a) $\mathcal{R}_{2}=0.9$, (b) $\mathcal{R}_{2}=1.5$, (c) $\mathcal{R}_{2}=2$, and (d) $\mathcal{R}_{2}=3$.

Our analytic results for the stabilities of equilibrira $E_{2}$ and $E_{3}$ (see Theorem 3 ) hold only for $d=0$ and $d^{\prime}=0$. Since the death rate $d^{\prime}$ from resistant-TB may be high, one would like to know if similar results hold when $d>0$ and $d^{\prime}>0$. Our numerical simulations suggest that this is the case. We first find from (2.5) a necessary condition under which 
the interior equilibium $E_{3}$ exists (see (17) in the Appendix). Using $\beta$ and $\beta^{*}$ as parameters we can establish a functional relationship between $\beta$ and $\beta^{*}$, that is, $\beta^{*}=h(\beta)$ where

$$
h(\beta)=\frac{1}{2 c}\left(D_{2}+\sqrt{D_{2}^{2}+4 D_{1} \beta}\right)
$$

with

$$
D_{1}=c R_{1 b}\left(1+\frac{1}{R_{2 a}}\right) \frac{\mu+k^{\prime}}{R_{2 a}}
$$

and

$$
D_{2}=\frac{\mu+k^{\prime}}{R_{2 a}}+\left(p r_{2} R_{1 b}-\left(\mu+k+r_{1}\right)\right)\left(1+\frac{1}{R_{2 a}}\right) .
$$

Then we have that $u^{*}=a-b v^{*}>0$ (a necessary condition for $E_{3}$ to exist, see (2.7) and (2.10)) iff

$$
\beta^{*}<h(\beta), \mathcal{R}_{1}>1
$$

By analogy with the proof for the case $d=d^{\prime}=0$ (see the proof of Theorem 3 in the Appendix) we would guess that $E_{3}$ exists and is l.a.s. if (3.1) holds and $E_{2}$ is l.a.s. if $\beta^{*}>h(\beta)$. Our guess is clearly supported by Fig. 5 .

The parameter values used in Fig. 5 are the same as those used in Fig. 4 except that $d=0.1$ and $d^{\prime}=0.5$. We choose $\bar{\beta}$ to be 13 (corresponding to $\overline{\mathcal{R}}_{1}=2.39$ ) and therefore $\bar{\beta}^{*}=h(\bar{\beta})=1.116$ (corresponding to $\overline{\mathcal{R}}_{2}=2.139$ ). We conclude that $E_{2}$ is l.a.s. if $\beta^{*}<1.116$ (or $\mathcal{R}_{2}<2.139$ ) and $E_{3}$ exists and is l.a.s. if $\beta^{*}>1.116$ (or $\mathcal{R}_{2}>2.139$ ). In Fig. 5 the values for $\beta^{*}$ are chosen to be (a) $\beta^{*}=0.4695\left(\mathcal{R}_{2}=0.9\right)$, (b) $\beta^{*}=0.78\left(\mathcal{R}_{2}=1.5\right)$, (c) $\beta^{*}=1.04\left(\mathcal{R}_{2}=2\right)$, and (d) $\beta^{*}=1.565\left(\mathcal{R}_{2}=3\right)$. 


\section{Discussion}

In this paper we introduced a basic model to study the dynamics of resistant TB. First we analyzed a one-strain TB model in order to understand its transmission dynamics in the absence of resistance. We proceeded to analyze a two-strain model for TB and resistantTB with the purpose of determining the role that lack of drug treatment compliance by TB patients plays on the maintenance of antibiotic resistant strains. To make the role of antibiotic resistance transparent, we first studied a special version of our two-strain model with two competing strains of TB: the typical strain plus a resistant strain that was not the result of antibiotic resistance. In this last situation, we found that co-existence is possible but rare while later we noticed that co-existence is almost certain when the second strain is the result of antibiotic resistance. Our mathematical analysis was based on quasisteady state approximations but our results were confirmed with the help of numerical simulations and the construction of bifurcation diagrams that support the plausability of our hypotheses.

A natural criticism of our basic model is that it did not take into account long and variable periods of latency - a key feature of TB. We have in fact looked at the effects of long and variable periods of latency (rather than the exponentially distributed delays used in this article) and we have found that their addition makes no difference in the qualitative dynamics of TB. However, we chose not to incorporate this analysis in this article as its emphasis is on the study of resistant TB. The incorporation of distributed delays would have obscured the objective of this article while making the mathematics quite ugly. The coupling of a two-strain model with different distributed delays for their latent periods is at this point under investigation. However, we are not very hopeful that we will be able to fully analyze this complex model. Nevertheless, we plan to publish some preliminary results in this direction in the near future.

A person infected with TB may develop active TB in a variety of ways. One possibility is that such a person may develop active TB as a consequence of exogenous-reinfection (i.e., acquiring a new infection from another infectious individual; Smith 1993). We have begun to look at the role that exogenous reinfection has on the transmission dynamics of common strains of TB. Our preliminary results seem to support our hypothesis that exogenous reinfection has a drastic effect on the qualitative dynamics of TB. More explicitly, the incorporation of exogenous reinfection into the basic TB model of Section 1 allows for the possibility of a subcritical bifurcation. That is, not only an endemic equilibium may occur at the critical value of the reproductive number $\mathcal{R}_{0}=1$ but our system can have multiple endemic equilibria for $\mathcal{R}_{0}<1$. This type of behavior has been observed in recent epidemiological models but in the context of sexually-transmitted diseases (see Hadeler and Castillo-Chavez 1995, to appear). Our analysis is almost complete and we plan to publish these results elsewhere in the near future. 
Finally, it is clear that mixing plays a key role in TB transmission. We are particularly interested in looking at the effects of age-dependent contact rates on TB dynamics. The formulation of models with age-dependent contact rates, even under the assumption of proportionate mixing, leads to hyperbolic systems of partial differential equations that are difficult to analyze. Nevertheless, we have managed to obtain some preliminary results and we plan to use them to study the role of the BCG vaccine on age-structured populations.

Acknowledgments This research was partially supported by NSF grant DEB-925370 (Presidential Faculty Fellowship Award) to Carlos Castillo-Chavez and by the U.S. Army Research Office through the Mathematical Science Institute of Cornell University (contract DAAL03-91-0027). 


\section{Appendix}

In this Appendix we provide the details of the proofs of Theorem 1,2 and 3 as well as the statements of needed preliminary results.

For a bounded real-valued function $f$ on $[0, \infty)$ we define

$$
f_{\infty}=\liminf _{t \rightarrow \infty} f(t), \quad f^{\infty}=\limsup _{t \rightarrow \infty} f(t)
$$

Lemma 1 (Thieme (1993)) Let $f:[0, \infty) \rightarrow \mathbf{R}$ be bounded and twice differentiable with bounded second derivative. Let $t_{n} \rightarrow \infty$ and $f\left(t_{n}\right)$ converge to $f^{\infty}$ or $f_{\infty}$ for $n \rightarrow \infty$. Then

$$
f^{\prime}\left(t_{n}\right) \rightarrow 0, \quad n \rightarrow \infty
$$

\section{Proof of Theorem 1:}

Let $\mathcal{R}_{0}<1$. Choose a sequence $t_{n} \rightarrow \infty$ such that

$$
I\left(t_{n}\right) \rightarrow I^{\infty}, \frac{d}{d t} I\left(t_{n}\right) \rightarrow 0 .
$$

Using the equation for $\frac{d}{d t} I$ in (1.3) and Lemma 1 we have

$$
I^{\infty} \leq \frac{k}{\mu+r_{2}+d} L^{\infty}
$$

Similarly, choosing a sequence $s_{n} \rightarrow \infty$ such that

$$
L\left(s_{n}\right) \rightarrow L^{\infty}, \frac{d}{d t} L\left(s_{n}\right) \rightarrow 0
$$

and using the equation for $\frac{d}{d t} \bar{L}$ in (1.3) we get

$$
\begin{aligned}
0 & \leq \beta c\left(\frac{S}{N}\right)^{\infty} I^{\infty}-\left(\mu+k+r_{1}\right) L^{\infty} \\
& \leq \beta c I^{\infty}-\left(\mu+k+r_{1}\right) L^{\infty} \\
& \leq\left(\mu+k+r_{1}\right)\left(\mathcal{R}_{0}-1\right) L^{\infty} .
\end{aligned}
$$

This implies that $L^{\infty} \leq 0\left(\right.$ since $\left.\mathcal{R}_{0}<1\right)$. But since $L_{\infty} \geq 0$, we have that $L^{\infty}=L_{\infty}=0$, and

$$
L(t) \rightarrow 0, \quad t \rightarrow \infty
$$

By (1) we also have that

$$
I(t) \rightarrow 0, \quad t \rightarrow \infty
$$


Using the $\frac{d}{d t} N$ equation (1.2) we get

$$
N_{\infty} \geq \frac{1}{\mu}\left(\Lambda-d I^{\infty}\right)=\frac{\Lambda}{\mu} .
$$

Since $N^{\infty} \leq \frac{\Lambda}{\mu}$, it follows that

$$
N_{\infty}=N^{\infty}=\frac{\Lambda}{\mu} .
$$

Hence $E^{0}$ is g.a.s. when $\mathcal{R}_{0}<1$.

Let $\mathcal{R}_{0}>1$. Noticing that

$$
\frac{W^{*}}{N^{*}}=\frac{1}{\mathcal{R}_{0}}
$$

we can write the Jacobian of (1.4) $J$ at $E^{*}$ as

$$
J=\left(\begin{array}{ccc}
-\mu & 0 & -d \\
a\left(\mathcal{R}_{0}-1\right) & -\left(a \mathcal{R}_{0}+\mu+r_{1}+k\right) & \frac{\beta c}{\mathcal{R}_{0}}-a \mathcal{R}_{0} \\
0 & k & -\left(\mu+d+r_{2}\right)
\end{array}\right)
$$

where

$$
a=\frac{\beta c}{\mathcal{R}_{0}} \frac{I^{*}}{N^{*}} .
$$

The characteristic equation is

$$
\lambda^{3}+A \lambda^{2}+B \lambda+C=0,
$$

where

$$
\begin{aligned}
& A=a \mathcal{R}_{0}+3 \mu+k+r_{1}+r_{2}+d, \\
& B=a \mathcal{R}_{0}\left(2 \mu+k+r_{2}+d\right)+\mu\left(2 \mu+k+r_{1}+r_{2}+d\right), \\
& C=\mu a \mathcal{R}_{0}\left(\mu+k+r_{2}+d\right)+k a d\left(\mathcal{R}_{0}-1\right) .
\end{aligned}
$$

It is clear that $A, C>0$. Since it can be easily checked that

$$
A B>C,
$$

then the Routh-Hurwitz stability conditions are satisfied. It follows that $E^{*}$ is l.a.s..

Proof of Theorem 2:

(a). Let $\mathcal{R}_{1}<1, \mathcal{R}_{2}<1$. By Lemma 1 and the $\frac{d}{d t} x_{2}, \frac{d}{d t} y_{2}$ equations in $(2.13)$ we have

$$
\begin{aligned}
& x_{2}^{\infty} \leq R_{1 b} x_{1}^{\infty}, \quad x_{2 \infty} \geq R_{1 b} x_{1 \infty} \\
& y_{2}^{\infty} \leq R_{2 a} y_{1}^{\infty}, \quad y_{2 \infty} \geq R_{2 a} y_{1 \infty}
\end{aligned}
$$


Using the $\frac{d}{d t} y_{1}$ equation in (2.13) and choosing $t_{n} \rightarrow \infty$ such that

$$
y_{1}\left(t_{n}\right) \rightarrow y_{1}^{\infty}, \quad \frac{d}{d t} y_{1}\left(t_{n}\right) \rightarrow 0, \quad t \rightarrow \infty,
$$

we get

$$
0 \leq-\left(\mu+k^{\prime}\right) y_{1}^{\infty}+\beta^{*} c\left(1-x_{2}-y_{1}-y_{2}\right)^{\infty} y_{2}^{\infty} .
$$

Using (2) and (2.14) we get

$$
0 \leq y_{1}^{\infty}\left(\mathcal{R}_{2}-1\right)
$$

and it is shown that $y_{1}^{\infty} \leq 0$ since $\mathcal{R}_{2}<1$. As $y_{1}^{\infty} \geq 0$, we have that $y_{1}^{\infty}=0$. The inequalities in (2) also imply that $y_{2}^{\infty}=0$. Similarly, using (2.14), the equation for $\frac{d}{d t} x_{1}$ in (2.13), and the inequalities in (2) we conclude that

$$
0 \leq x_{2}^{\infty}\left(\mathcal{R}_{1}-1\right)
$$

Since $\mathcal{R}_{1}<1$, we have that $x_{2}^{\infty} \leq 0$. It follows that $x_{1}^{\infty}=x_{2}^{\infty}=0$. Hence

$$
\lim _{t \rightarrow \infty} x_{1}(t)=\lim _{t \rightarrow \infty} x_{2}(t)=\lim _{t \rightarrow \infty} y_{1}(t)=\lim _{t \rightarrow \infty} y_{2}(t)=0 .
$$

(b). Let $\mathcal{R}_{1}>1$. Denote the corresponding equilibrium $E_{4}$ for $(2.13)$ by $\left(\bar{x}_{1}, \bar{x}_{2}, \bar{y}_{1}, \bar{y}_{2}\right)$. Then

$$
\left(\bar{x}_{1}, \bar{x}_{2}, \bar{y}_{1}, \bar{y}_{2}\right)=\left(\frac{e\left(\mathcal{R}_{1}-1\right)}{R_{1 b}}, e\left(\mathcal{R}_{1}-1\right), 0,0\right),
$$

where $e$ is given by (2.12). The eigenvalues of the Jacobian of (2.13) at $E_{4}$ are given by the following two equations (keeping in mind that $q=0$ ):

$$
\begin{aligned}
& \lambda^{2}+\left(\beta c \bar{x}_{2}+2 \mu+k+r_{1}+r_{2}\right) \lambda+\beta c \bar{x}_{2}\left(\mu+r_{2}+k\right)=0, \\
& \lambda^{2}+\left(2 \mu+k^{\prime}\right) \lambda+\mu\left(\mu+k^{\prime}\right)+k^{\prime} \beta^{*} c\left(\bar{x}_{2}-1\right)=0 .
\end{aligned}
$$

The first equation in (3) always has two eigenvalues with negative real pärts. Both roots of the second equation in (3) have negative real parts if and only if the constant term is positive which is equivalent to

$$
\mathcal{R}_{2}<\frac{1}{1+\frac{\left(1-\mathcal{R}_{1}\right)}{R_{1 a}\left(1+R_{1 b}\right)}}
$$

Let

$$
f\left(\mathcal{R}_{1}\right)=\frac{1}{1+\frac{\left(1-\mathcal{R}_{1}\right)}{R_{1 a}\left(1+R_{1 b}\right)}},
$$

it follows that $E_{4}$ is l.a.s. if $\mathcal{R}_{2}<f\left(\mathcal{R}_{1}\right)$. If $\mathcal{R}_{2}>f\left(\mathcal{R}_{1}\right)$, then the second equation in (3) has one root with positive real part, and hence $E_{4}$ is unstable. It is easy to show that $f\left(\mathcal{R}_{1}\right)>1$ for all $\mathcal{R}_{1}>1$ and $f(1)=1$. 
(c). Let $\mathcal{R}_{2}>1$. Note that the corresponding equilibrium $E_{2}$ now is

$$
\left(\tilde{x}_{1}, \tilde{x}_{2}, \tilde{y}_{1}, \tilde{y}_{2}\right)=\left(0,0,\left(1-\frac{1}{\mathcal{R}_{2}}\right) \frac{\mu}{\mu+k^{\prime}},\left(1-\frac{1}{\mathcal{R}_{2}}\right) \frac{k^{\prime}}{\mu+k^{\prime}}\right) .
$$

The eigenvalues $w$ of the Jacobian of (2.13) at the corresponding equilibrium $E_{2}$ are given by the following two equations:

$$
\begin{aligned}
& w^{2}+\left(\mu+k+r_{1}+\mu \mathcal{R}_{2}+r_{2}\right) w+\alpha=0 \\
& w^{2}+\left(2 \mu+k^{\prime}+\mu\left(\mathcal{R}_{2}-1\right)\right) w+\mu\left(\mu+k^{\prime}\right)\left(\mathcal{R}_{2}-1\right)=0
\end{aligned}
$$

where

$$
\alpha=\left(\mu+k+r_{1}\right)\left(\mu+r_{2}\right)\left(1-\mathcal{R}_{1}\right)+\mu\left(\mu+r_{2}\right)\left(\mathcal{R}_{2}-1\right)+k \beta c\left(1-\frac{1}{\mathcal{R}_{2}}\right) .
$$

In the second equation of (5), since the coefficients are all positive there are always two roots with negative real parts. In the first equation of (5), both roots have negative real parts iff $\alpha>0$ which is true if $\mathcal{R}_{1}<1$ or if $\mathcal{R}_{1}>1$ and

$$
q_{1 a} \mathcal{R}_{2}^{2}+\left(1-\mathcal{R}_{1}-q_{1 a}+R_{1 a} R_{1 b}\right) \mathcal{R}_{2}-R_{1 a} R_{1 b}>0
$$

As a quadratic function of $\mathcal{R}_{2}$, the left hand side of (6) has exactly one positive root which we denote by $R_{2}^{+}=g\left(\mathcal{R}_{1}\right)$. Then $\alpha>0$ iff $\mathcal{R}_{1}<1$ or

$$
\mathcal{R}_{1}>1 \text { and } \mathcal{R}_{2}>g\left(\mathcal{R}_{1}\right)
$$

where

$$
g\left(\mathcal{R}_{1}\right)=\frac{1}{2 q_{1 a}}\left(\mathcal{R}_{1}-1+q_{1 a}-R_{1 a} R_{1 b}+\sqrt{\left(\mathcal{R}_{1}-1+q_{1 a}-R_{1 a} R_{1 b}\right)^{2}+4 q_{1 a} R_{1 a} R_{1 b}}\right) .
$$

To check that $g\left(\mathcal{R}_{1}\right)>1$, let $F(z)$ be the function defined by the left hand side of $(6)$ (as a function of $\mathcal{R}_{2}$ ). Then $F\left(R_{2}^{+}\right)=0$, and $z<R_{2}^{+}$iff $F(z)<F\left(R_{2}^{+}\right)$or $F(z)<0$. If $\mathcal{R}_{1}>1$, then it is easy to see that $F(1)<0$. Hence $1<R_{2}^{+}$, i.e., $g\left(\mathcal{R}_{1}\right)>1$. Then we conclude that $E_{2}$ is l.a.s. if $\mathcal{R}_{1}<1$ or if (7) holds.

If $\mathcal{R}_{1}>1$ and $\mathcal{R}_{2}<g\left(\mathcal{R}_{1}\right)$, then $\alpha<0$ and the first equation in (5) has one root with positive real part, and hence $E_{2}$ is unstable.

Note that

$$
\begin{aligned}
F\left(f\left(\mathcal{R}_{1}\right)\right) & =R_{1 a}\left(\mathcal{R}_{1}-1\right)\left(q_{1 a}+q_{1 a} R_{1 b}+\mathcal{R}_{1}-1-R_{1 a}-R_{1 a} R_{1 b}\right) \\
& \leq \frac{R_{1 a}\left(1-\mathcal{R}_{1}\right)}{(\mu+k+r 1)(\mu+r 2)}\left(k r_{2}(1+p)+\left(r_{1}+\beta c\right)\left(\mu+r_{2}\right)\right) \\
& \leq 0
\end{aligned}
$$


for all $\mathcal{R}_{1}>1$. It follows that $g\left(\mathcal{R}_{1}\right)>f\left(\mathcal{R}_{1}\right)$ for all $\mathcal{R}_{1}>1$. It is easy to see that $g(1)=1$.

(d). $E_{3}$ is feasible iff $u^{*}>0$ and $v^{*}>0$ where $u^{*}$ and $v^{*}$ are the solutions of equations (2.7) and (2.10) with $C=0$ (since $q=0$ ). Note that

$$
v^{*}=-\frac{B}{A} .
$$

The equivalent condition for $v^{*}>0$ is that $A$ and $B$ have opposite signs which turns out to be if the following condition is satisfied:

$$
f\left(\mathcal{R}_{1}\right)<\mathcal{R}_{2}<\frac{\beta c}{\mu}
$$

Here we have used the fact that $f\left(\mathcal{R}_{1}\right)<\frac{\beta c}{\mu}$. Expression (9) implies that $A>0$ and $B<0$. By (2.7) the equivalent condition for $u^{*}>0$ is that $a / b>v^{*}$ which is satisfied when the inequality (6) or (7) change direction, i.e., when

$$
\mathcal{R}_{2}<g\left(\mathcal{R}_{1}\right)
$$

It can be checked that $g\left(\mathcal{R}_{1}\right)=R_{2}^{+}<\frac{\beta c}{\mu}$. Then (9) and (10) imply that $f\left(\mathcal{R}_{1}\right)<\mathcal{R}_{2}<$ $g\left(\mathcal{R}_{1}\right)$, which also implies by part (c) that $\mathcal{R}_{1}>1$. It follows that $E_{3}$ exists iff

$$
\mathcal{R}_{1}>1, \quad f\left(\mathcal{R}_{1}\right)<\mathcal{R}_{2}<g\left(\mathcal{R}_{1}\right) .
$$

At the quasi-steady state, $x_{2}=R_{1 b} x_{1}, y_{2}=R_{2 a} y_{1}$, System (2.13) reduces to a two dimensional system:

$$
\begin{aligned}
& \frac{d}{d t} x_{1}=\left(\mu+k+r_{1}\right)\left(\mathcal{R}_{1}-1\right) x_{1}-\beta c\left(R_{1 b}+R_{1 b}^{2}\right) x_{1}^{2}-\left(\beta c R_{1 b}\left(1+R_{2 a}\right)+\beta^{*} c R_{2 a}\right) x_{1} y_{1} \\
& \frac{d}{d t} y_{1}=\left(\mu+k^{\prime}\right) \mathcal{R}_{2}\left(\left(1-\frac{1}{\mathcal{R}_{\tilde{\Sigma}}}\right) y_{1}-R_{1 b} x_{1} y_{1}-\left(1+R_{2 a}\right) y_{1}^{2}\right) .
\end{aligned}
$$

The positive equilibrium of (12) corresponding to $E_{3}$ is

$$
x_{1}=\frac{u^{*}}{R_{1 b}}, \quad y_{1}=\frac{v^{*}}{R_{2 a}} .
$$

One can show that the the Jacobian of (12) at (13) has two eigenvalues with negative real part(s) iff

$$
\mathcal{R}_{2}<\frac{\beta c}{\mu} .
$$

Notice that $\mathcal{R}_{2}<\mathcal{R}_{2}^{+}<\frac{\beta c}{\mu}$. The local stability follows. 
This finishes the proof.

Remark: Functions $f\left(\mathcal{R}_{1}\right)$ and $f_{2}\left(\mathcal{R}_{1}\right)$ can be written in different forms depending on which parameter(s) we want to vary. For example if we want $\beta$ to be a varying parameter with other parameters fixed, we can write $f$ and $g$ as the following (noticing that $\mathcal{R}_{1}$ is a function of $\beta$ ):

$$
\begin{aligned}
& f\left(\mathcal{R}_{1}(\beta)\right)=\frac{1}{1+\left(1-\mathcal{R}_{1}(\beta)\right)\left(\left(1+R_{1 b}\right)\left(\frac{\mathcal{R}_{1}(\beta)}{R_{1 b}}-\frac{p r_{2}}{\mu+k+r_{1}}\right)\right)^{-1}} \\
& g\left(\mathcal{R}_{1}(\beta)\right)=\frac{1}{2 q_{1 a}}\left(-l+\sqrt{l^{2}+4 q_{1 a}\left(\mathcal{R}_{1}(\beta)-n\right)}\right)
\end{aligned}
$$

where

$$
\begin{aligned}
l & =\frac{\mu\left(k+r_{1}\right)+r_{1} r_{2}+(1-p) k r_{2}}{\left(\mu+k+r_{1}\right)\left(\mu+r_{2}\right)} \\
n & =\frac{k p r_{2}}{\left(\mu+k+r_{1}\right)\left(\mu+r_{2}\right)}
\end{aligned}
$$

\section{Proof of Theorem 3:}

(i) is true since the proof of part (a) in Theorem 2 is valid for any $q \geq 0$.

Notice that $q$ does not appear in both the expression of $E_{2}$ and the Jacobian of (2.13) at $E_{2}$. Also $q$ is not involved in the proof of theorem 2 (c). (ii) follows immediately.

For part (iii), recall that $v^{*}$ is the solution of (2.9). As $C<0$ there exists a unique positive real root $v^{*}$ iff $A>0$, which (after some algebra) is equivalent to

$$
\mathcal{R}_{2}<\frac{\beta c}{\mu}
$$

As $b v^{*}>0$, a necessary condition for $u^{*}>0$ is that $a>0$, i.e, that

$$
\mathcal{R}_{1}>1
$$

Let $G(z)=A z^{2}+B z+C$ (a function given by the left hand side of (2.9) in Section 2), then $G\left(v^{*}\right)=0$. Since $G$ is a parabola with $A>0$, and since $v^{*}$ is the only positive root, $u^{*}>0\left(\right.$ or $\left.a / b>v^{*}\right)$ iff

$$
G\left(\frac{a}{b}\right)>G\left(v^{*}\right)=0
$$

This is equivalent to

$$
\left(1+\frac{1}{R_{2 a}}\right) \frac{a}{b}+\frac{1}{\mathcal{R}_{2}}-1>0
$$


After some algebra we can see that (17) is equivalent to

$$
q_{1 a} \mathcal{R}_{2}^{2}+\left(1-\mathcal{R}_{1}-q_{1 a}+R_{1 a} R_{1 b}\right) \mathcal{R}_{2}-R_{1 a} R_{1 b}<0
$$

Note that (18) has just the opposite direction as that of the inequality (6) which implies that

$$
\mathcal{R}_{2}<g\left(\mathcal{R}_{1}\right) \text {. }
$$

Hence $u^{*}>0$ iff (16) and (19) hold. Also noticing that $g\left(\mathcal{R}_{1}\right)=R_{2}^{+}<\frac{\beta c}{\mu}$, we see that (19) implies (15), i.e., $u^{*}>0$ implies that $v^{*}>0$ exists and is unique. Hence $E_{3}$ exists iff $\mathcal{R}_{1}>1$ and $\mathcal{R}_{2}<g\left(\mathcal{R}_{1}\right)$. The proof of the local asymptotical stability is similar to the one in Theorem 2 (d).

This finishes the proof. 


\section{References}

Anderson, R.M.: Population dynamics of infectious disease. London New York: Chapman and Hall 1982

Anderson, R.M., May, R.M.: Population biology of infectious diseases. Springer-Verlag Berlin Heidelberg New York: 1982

Anderson, R.M., May, R.M.: Infectious Diseases of Humans. Oxford New York Tokyo: Oxford University Press 1991

Ayvazian, L.F.: History of tuberculosis. Reichman, L.B.; Hershfield, E.S. (eds.) Tuberculosis: A Comprehensive International Approach. pp.1-20 New York 1993.

Bloom, B.R.: Tuberculosis: Pathogenesis, Protection, and Control. Washington, D.C. 1994.

Blower, S.: (ed.) Abstract from Mathematical Modeling of Tuberculosis. (1995)

Castillo-Chavez,C., Hethcote, H.W., Andreason, V. Levin, S.A., Liu, W.: Cross-immunity in the dynamics of homogeneous and geterogeneous populations. In: Gross, L., Hallam, T.G., Levin, S.A. (eds.) Mathematical ecology. Proceedings, Autumn Course Research Seminars, Trieste 1986, pp.303-316. Singapore: World Scientific Publ. Co. 1988

Castillo-Chavez, C., Hethcote, H.W., Andreason, V. Levin, S.A., Liu, W.: Epidemiological models with age structure, proportionate mixing; and cross-immunity. J. Math. Biol. 27, 233-258 (1989)

Castillo-Chavez, C., Thieme, R.H.: Asymptotically autonomous epidemic models. In: Proc. Third Intern. Conf. on Mathematical Population Dynamics (O. Arino and M. Kimmel, eds.) (in press)

Dietz, K.: Epidemiologic interference of virus populations. J. Math. Biol. 8, 291-300 (1979)

Dietz, K., Schenzle, D.: Proportionate mixing models for age-dependent-infection transmission. J.Math. Biol. 22, 117-120 (1985)

Feng, Z., Thieme, R.H.: Multi-annual outbreaks of childhood disease revisited: the impact of isolation. (to apper) Math. Biosci. (1995)

Feng, Z. (thesis): A mathematical model for the dynamics of childhood disease under the impact of isolation. Arizona State University (1994)

Hadeler, K.P., Castillo-Chavez,C.: A core group model for disease transmission. (to apper) Math. Biosci. (1995)

Hethcote, H.W.: Qualitative analysis for communicable disease models. Math. Biosci. 28, 335-356 (1976)

Hethcote, H.W., Stech, H.W., van den Driessche, P.: Periodicity and stability in epidemic models: a survey. In: Busenberg, S., Cooke, K.L. (eds.) Differential equations and 
applications in ecology, epidemics and population problems, pp. 65-82. New York: Academic Press 1981

Hethcote, H.W., Van Ark, J.W.: Epidemiological models for heterogenous popoulations: proportionate mixing, parameter estimation and immunization programs. Math. Biosci., 84, 85-118 (1987)

Hopewell, P.C.: Overview of clinical tuberculosis. Bloom B.R. (ed.) Tuberculosis: Pathogenesis, protection, and contro, pp. 25-46 (1994)

Kent, J.H.: The epidemiology of multi-grug resistant tuberculosis in the United States. Medical clinics of North America. 77, 1-6 (1991)

Kochi, A.: The Global Tuberculosis Situation and the New Control Strategy of the World Health Organization. Tubercle 72, 1-6 (1991)

Miller, Bess.: Preventive Therapy for Tuberculosis. Medical clinics of North America. 77, 1263-1275 (1993)

MMWR: Morbidity and mortality weekly report. 44 No.8 137-154 (1995)

Press release WHO /89: TB Epidemic Outpacing New Drug Development. 17 Nov. 1994

Reichman, L.B.; Hershfield, E.S.: Tuberculosis: A Comprehensive International Approach. New York 1993.

Salyers, A.A.; Whitt, D.D.: Bacterial Pathogensis. Washington, D.C. 1994.

Schenzle, D.: An age-structured model of pre- and post-vaccination measles transmission. IMA J. Math. Appl. Med. Biol. 1, 169-191 (1984)

Smith, P.G., Moss, A.R.: Epidemiology of tuberculosis. Bloom B.R. (ed.) Tuberculosis: Pathogenesis, protection, and contro, pp. 47-59 (1994)

Styblo, K.: Selected Papers: Epidemiology of Tuberculosis. Royal Netherlands Tuberculosis Association. 24, 55-62 (1991)

Kolata, G.: First documented case of TB passed on airliner is reported by the U.S.. New York Times. March 3, 1995

Thieme, R.H.: Persistence under relaxed point-dissipativity (with applications to an endemic modei). SIÂM J. Math. Anal. 24, 407-435 (1993)

Thieme, R.H.: Convergence results and a Poincaré-Bendixson tricho-tomy for asymptotical autonomous differential equations. J. Math. Biol. 30, 755-763 (1992)

Thieme, R.H.: Asymptotical autonomous differential equations in the plane. Rocky Mountain J. Math. 24 351-380 (1994) 


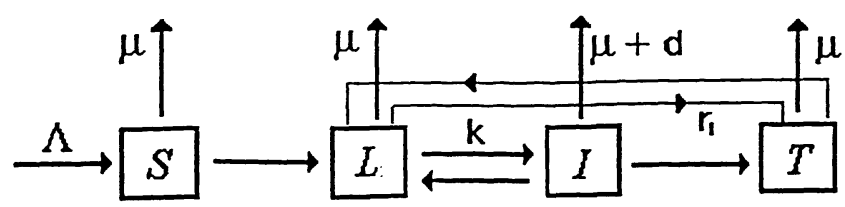

Fig.1.a. A diagram for one strain TB disease transmission. $S$ stands for susceptible, $L$ - exposed (latent), $I$ - infectious, $T$ treated. $\Lambda$ is the recruitment rate, $\mu$ is the per capita death rate, $d$ is the disease-induced death rate (per capita), $r_{1}$ and $r_{2}$ denote the treatment rates for latent and infectious individuals, respectively. Individuals in $S$ and $T$ classes can be infected only through contacts with infectious individuals.

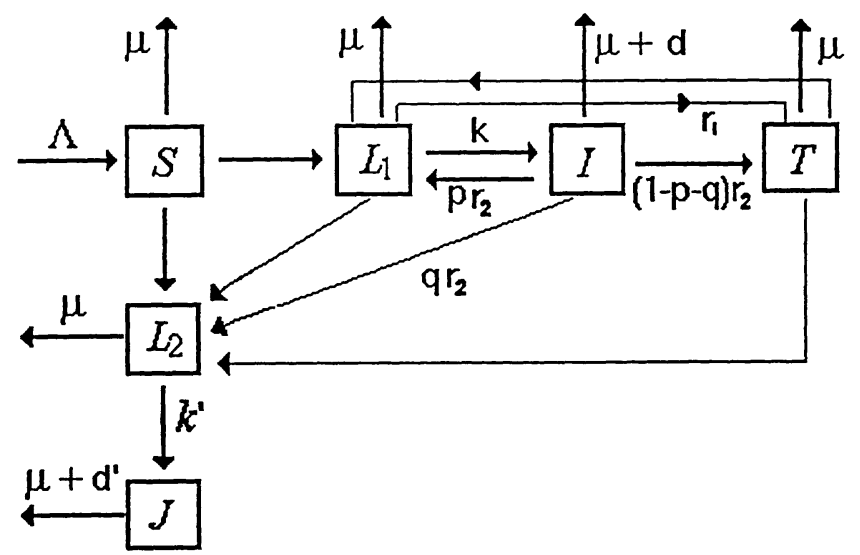

Fig.1.b. A diagram for two-strains TB transmission. $L_{1}$ and $L_{2}$ denote individuals exposed to typical TB and antibiotic resistant TB, respectively. $J$ stands for infectious individuals with resistant TB. $p+q$ is the proportion of those treated infectious individuals who did not complete their treatment. The proportion $p$ modifies the rate that departs from the latent class, and hence $q r_{2} I$ gives the rate at which individuals develop resistant-TB because they did not complete the treatment of active TB. $p \geq 0, q \geq 0$ and $p+q \leq 1 . \Lambda, \mu, d, r_{1}, r_{2}$ have the same meanings as in Fig.1.a and $d^{\prime}$ is the disease (resistant TB) induced death rate. 

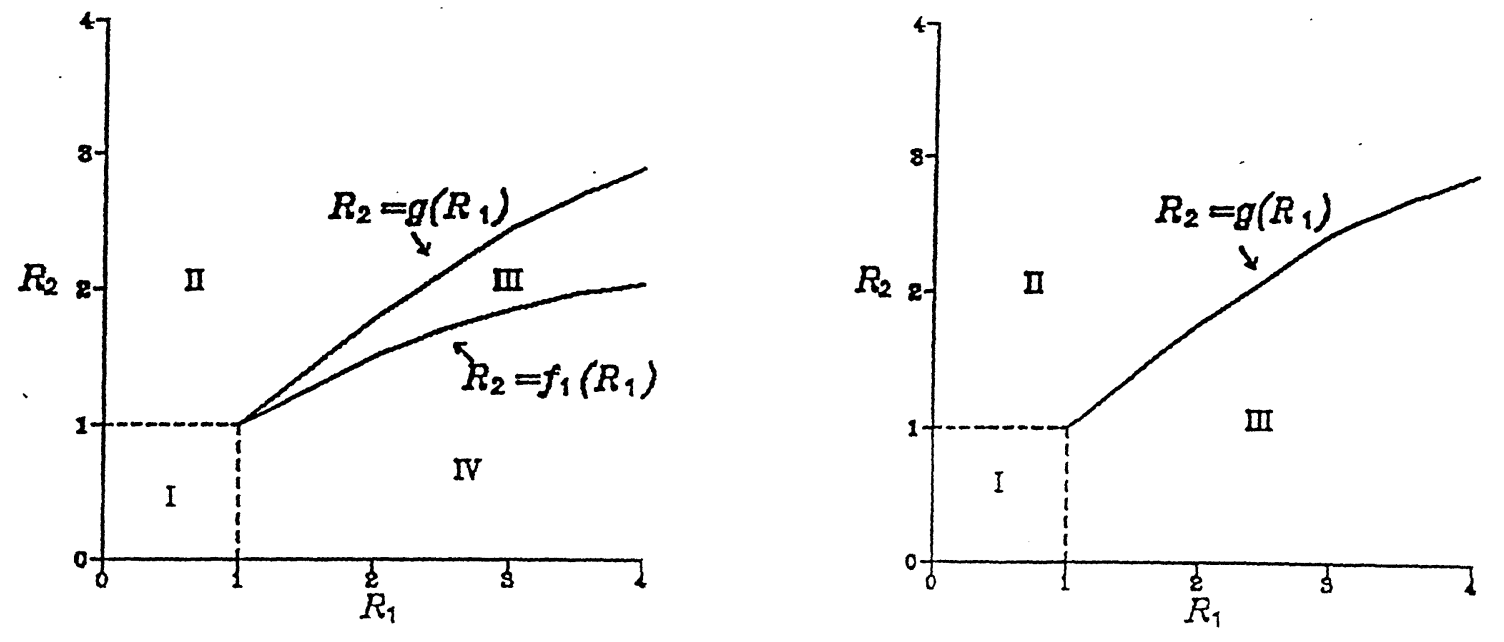

Fig.2.a. A bifurcation diagram for the system (2.5) in the case $q=0$. There are four regions I, II, III, and IV in the parameter space $\left(\mathcal{R}_{1}, \mathcal{R}_{2}\right)$. In the region I $E_{1}$ is a global atractor and other equilibria are unstable when they exist. In regions II and IV $E_{3}$ does not exist and $E_{2}$ and $E_{4}$ are l.a.s., respectively. In the region III $E_{3}$ exists and is l.a.s..

Fig.2.b. A bifurcation diagram for the system (2.5) in the case $q>0$. There are three regions I, II and III in the parameter space $\left(\mathcal{R}_{1}, \mathcal{R}_{2}\right)\left(E_{4}\right.$ does not exist.), and they correspond to stabilities of $E_{1}, E_{2}$, and $E_{3}$, respectively. 

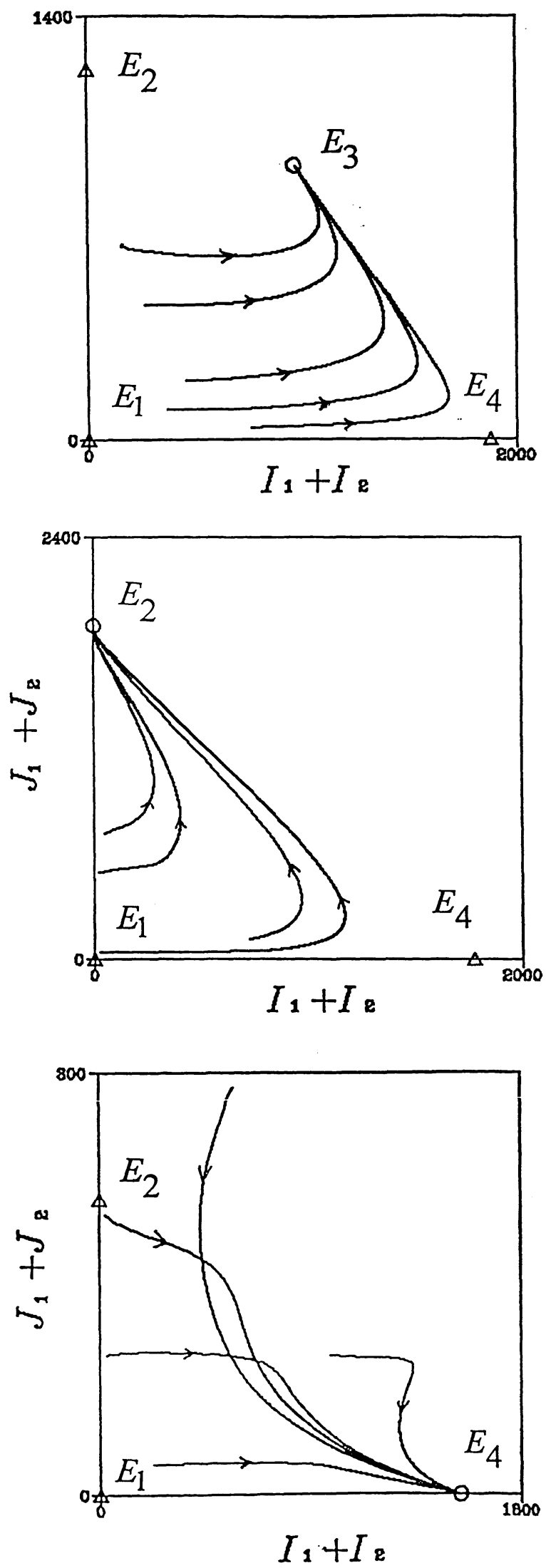

Fig.3. Phase portraits of solutions to (2.5). The parameter values for all three graphs are chosen to be: $\mu_{\bar{z}}=0.143, \beta=$ $13, c=1, k=1, q=0, p=0.5, r_{1}=$ $1, r_{2}=2, k^{\prime}=1, \Lambda=35, d=d^{\prime}=0$. This choice of parameters gives a fixed value $\mathcal{R}_{1}=3.45$. In (a) $\mathcal{R}_{2}=2$ and hence $\left(\mathcal{R}_{1}, \mathcal{R}_{2}\right) \in$ III. In (b) $\mathcal{R}_{2}=4.5$ and hence $\left(\mathcal{R}_{1}, \mathcal{R}_{2}\right) \in$ II. In (c) $\mathcal{R}_{2}=1.2$ and hence $\left(\mathcal{R}_{1}, \mathcal{R}_{2}\right) \in I V$. Circle " $O$ " indicates a stable equilibrium, and triangle " $\triangle$ " indicates an unstable equilibrium. (Graphs are given by $\mathrm{Phsplan}$ ). 



Fig.4. Plots of fractions of infected and infectious population versus time in the case $q>0, d=d^{\prime}=0$. Parameters for all graphs are chosen to be: $\mu=0.143, \beta=13, c=1, k=0.5, q=0.01, p=$ $0.4, r_{1}=2, r_{2}=1, k^{\prime}=1, \Lambda=500, d=d^{\prime}=0$.

In (a) $\mathcal{R}_{2}=0.9$ and hence $\left(\mathcal{R}_{1}, \mathcal{R}_{2}\right) \in I I I$. In (b) $\mathcal{R}_{2}=1.5$ and $\left(\mathcal{R}_{1}, \mathcal{R}_{2}\right) \in I I I$. In (c) $\mathcal{R}_{2}=2$ and $\left(\mathcal{R}_{1}, \mathcal{R}_{2}\right) \in I I I$. In (d) $\mathcal{R}_{2}=3$ and $\left(\mathcal{R}_{1}, \mathcal{R}_{2}\right) \in I V$. 



Fig.5. Plots of fractions of infected and infectious population versus time in the case $q>0, d>0, d^{\prime}>0$. Parameters for all graphs are chosen to be: $\mu=0.143, \beta=13, c=1, k=0.5, q=$ $0.01, p=0.4, r_{1}=2, r_{2}=1, k^{\prime}=1, \Lambda=500, d=0.1, d^{\prime}=0.5$.

In (a) $\mathcal{R}_{2}=0.9$ and hence $\left(\mathcal{R}_{1}, \mathcal{R}_{2}\right) \in I I I$. In (b) $\mathcal{R}_{2}=1.5$ and $\left(\mathcal{R}_{1}, \mathcal{R}_{2}\right) \in I I I$. In (c) $\mathcal{R}_{2}=2$ and $\left(\mathcal{R}_{1}, \mathcal{R}_{2}\right) \in I I I$. In (d) $\mathcal{R}_{2}=3$ and $\left(\mathcal{R}_{1}, \mathcal{R}_{2}\right) \in I V$. 